\title{
User-Guided Rendering of Audio Objects Using an Interactive Genetic Algorithm
}

\author{
ALEX WILSON, AES Associate Member, AND BRUNO M. FAZENDA, AES Member \\ (alex.wilson199@gmail.com) \\ (b.m.fazenda@salford.ac.uk) \\ Acoustics Research Centre, University of Salford, Salford, M5 4WT, UK
}

\begin{abstract}
Object-based audio allows for personalization of content, perhaps to improve accessibility or to increase quality of experience more generally. This paper describes the design and evaluation of an interactive audio renderer, which is used to optimize an audio mix based on the feedback of the listener. A panel of 14 trained participants were recruited to trial the system. The range of audio mixes produced using the proposed system was comparable to the range of mixes achieved using a traditional fader-based mixing interface. Evaluation using the System Usability Scale showed a low level of physical and mental burden, making this a suitable interface for users with impairments, such as to vision and/or mobility.
\end{abstract}

\section{INTRODUCTION}

One of the advantages of object-based audio/broadcast over traditional channel-based delivery is that it allows for the rendering of personalized content upon being delivered to the audience [1]. The methods by which personalization are achieved often require an in-depth understanding of the problem domain. For example, automatic mixing of music has built on developments in music information retrieval [2], audio engineering practice [3, 4], and the emotional response to music [5]. Meanwhile, in broadcast domains, the clarity and intelligibility of dialogue is often crucially important [6]. The interaction between speech signals and other audio and visual objects, such as music and sound effects, and the overall effect on the audience, must be well-understood for a variety of audience groups [7].

This paper describes a system that is designed to render an optimal audio mix by learning the preferences of the user and writing/rewriting object metadata (such as level and panning). While the focus is on music signals, the scope of the work extends to linear mixing of audio signals in any domain. The example provided will assume no existing metadata, in order to demonstrate the flexibility of the system in adapting to user requirements, using a "listener-as-an-object" paradigm.

\section{LITERATURE REVIEW}

The perceived quality of an audio mix is dependent on both subjective impressions and objective measures of the signal [8]. Additionally, there is some evidence to suggest that listeners can regularly perceive the different styles of mix engineers $[3,9]$. These observations suggest that it is important to allow the user to guide a rendering system to some personal optimum. We propose the following requirements for such a system.

1. Explore a solution space that is representative of the mixing process;

2. Acknowledge that more than one optimal solution may exist;

3. That the optimal solution(s) may vary from user to user, whether based on specific accessibility requirements or hedonic preference.

Evolutionary computing is a framework that suits these requirements. A literature review on the use of $\mathrm{EC}$ in audio applications, and the argument for it's use in an interactive audio renderer, can be found in earlier work by the authors [10]. An appropriate solution space has been proposed (a space of mixes, referred to herein as a "mix-space") [11], as well as a method of generating a population of mixes within it [12], which is an early step in an evolutionary algorithm. The work in this paper follows on immediately from [12] and describes a method of finding personally-optimal mixes within this space.

\section{DESIGN}

The flowchart in Fig. 1 illustrates the design of an audio mixing application based on an interactive genetic algorithm (IGA). The important steps in this flowchart are each described in the following subsections. The aim of the proposed system is to obtain an optimal gain vector, containing 


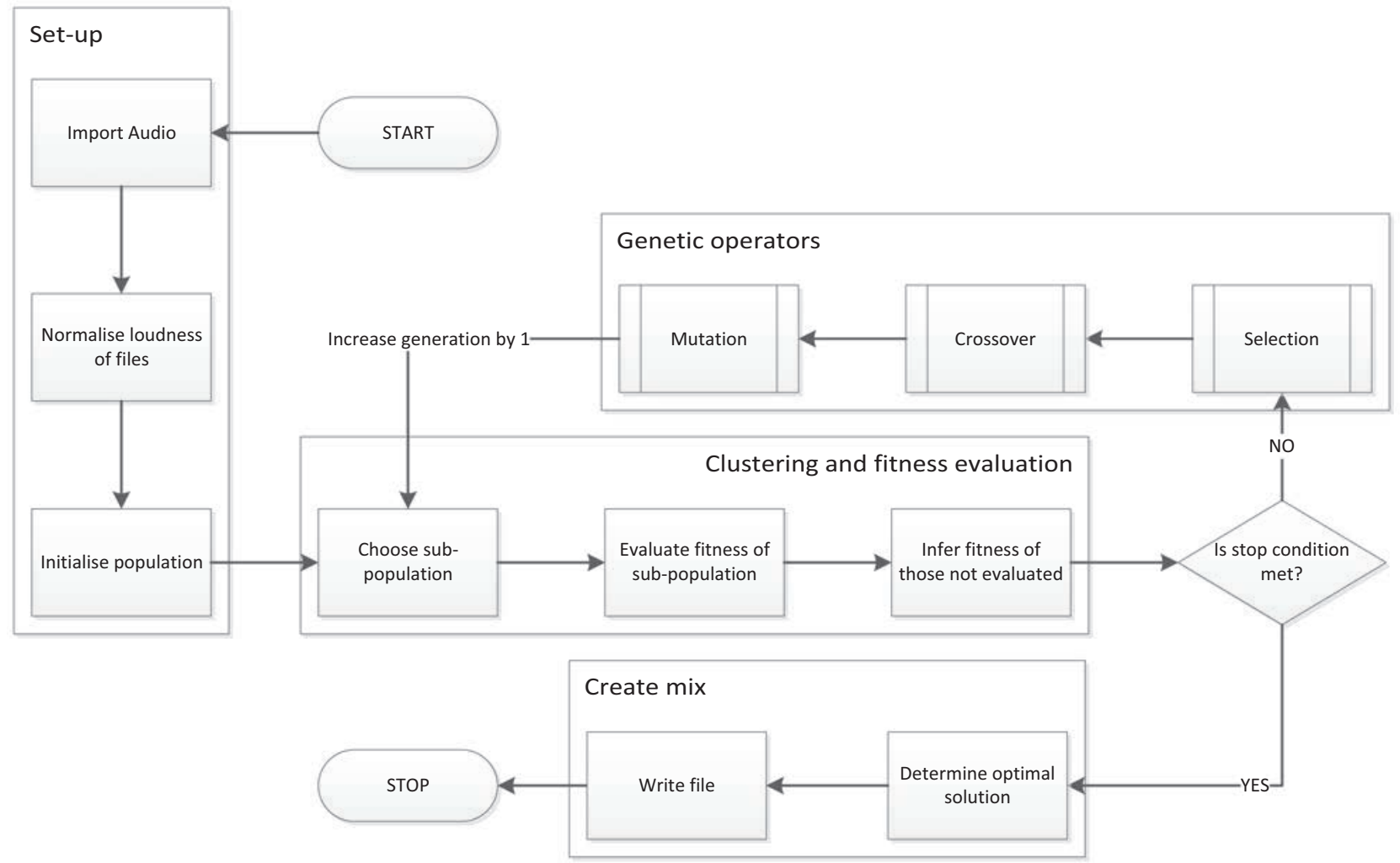

Fig. 1. Flowchart illustrating the design of the system.

one element per object, which is used to create a static mix of the audio objects. Additional object metadata, such as position and equalization parameters, are not considered here although they are theoretically equivalent [12] and object-panning in stereo has been implemented in previous work by the first author [9].

\subsection{Set-Up}

The test audio used in trials of the developed system is of the following form: a total of six tracks where each is a single-channel.WAV file, PCM encoded at a sampling rate of $44.1 \mathrm{kHz}$, and a bit depth of 16-bits. The six tracks represent vocals, guitar, bass guitar, snare drum, kick drum, and a single overhead microphone. This precise ordering of tracks ensured that, using the proposed framework [11, 12], the five parameters of the mix $\left(\phi_{1}, \ldots, 5\right)$ have a clear meaning: $\phi_{1}$ indicates the balance of the vocal to the backing tracks, $\phi_{2}$ is the balance of the guitar to the "rhythm section" of bass and drums, $\phi_{3}$ represents the balance of bass to drums, and so on.

Considering the relatively narrowband content of the individual tracks in a multitrack session, loudness was normalized according to a modified form of ITU-BS.1770 [13]. This ensures that the loudness of each track in a mix can be retrieved directly from the gain vector and that all points in the mix-space have the same perceived loudness [12].

The initial population of mixes was sampled from a vonMises-Fisher (vMF) distribution, with mean vector $\mu$ and concentration parameter $\kappa$ [12]. The vMF distribution can be considered the spherical equivalent of a Gaussian distribution. Being points of the surface of a unit hypersphere ensures that the $\ell_{2}$ norm of the gain vector is equal to 1 . This has the advantage that each mix is presented at roughly equal loudness while also having sufficient headroom to avoid clipping. The mean vector $\mu$ represents the initial guess for the desired mix. This could come from literature or from a previous optimization session. In this paper we begin with no assumptions as to what mix would be ideal - a uniform distribution was obtained using arbitrary $\mu$ and with $\kappa=0$.

\subsection{Clustering and Fitness Evaluation}

With a large population, evaluating each mix will be fatiguing for a user. Rather than directly evaluate the entire population, one need only rate a sub-population of size $c$, reducing user burden. To achieve this the total population is divided into $c$ clusters and a single representative mix is taken from each cluster. After a series of tests (described in [9]) the points were clustered in the gain-space $\left(\mathbb{R}^{n}\right.$, where $n$ is the number of tracks) using spherical $k$-means clustering, where the distance metric is the Cosine distance [14]. An example is shown in Fig. 2.

Once the sub-population is determined, the fitness of each solution (each mix in this case) is evaluated. How this is achieved depends on the fitness function. In a standard GA approach, this function must be well-defined [15]. In IGA applications, the fitness is evaluated by the user [16] but can be augmented by an objective function, such as those defined in [17], for example. In this system, each mix in the sub-population is played back to the user and directly evaluated on a chosen scale, such as preference. Other scales may be used depending on the task. 
Table 1. Settings used in the evaluation of the IGA mixer

\begin{tabular}{lll}
\hline Parameter & \multicolumn{1}{c}{ Description } & Value \\
\hline$n$ & Number of audio tracks being mixed & 6 \\
$d$ & Number of variables/dimensions in solution space & $n-1$ \\
Population size & Number of candidate solutions per generation & 100 \\
$c$ & Number of solutions to be auditioned/evaluated in each generation & 5 \\
$q$ & Number of bits used to represent the value of each variable & 7 \\
Elite fraction & Proportion of children generated as clones of fittest parents & 0.05 \\
Crossover fraction & Proportion of children generated by crossover of two parents & 0.85 \\
Mutation fraction & Amount of bits to be mutated in the remaining children & $\lfloor(q \times d) / 3\rfloor$ \\
Stop condition & Condition which, when met, causes evolution to cease & 10 generations \\
\hline
\end{tabular}
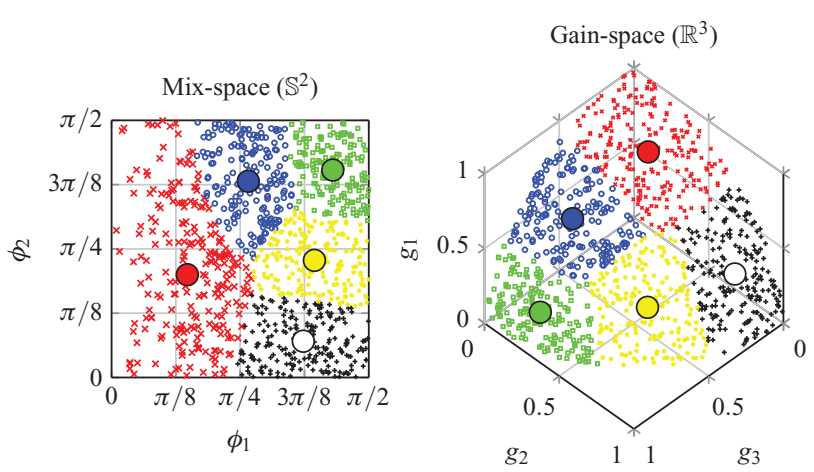

Fig. 2. 1,000 solutions to a 3-track mixing problem, clustered using spherical $k$-means. The solutions closest to each of the cluster centroids form the sub-population to be evaluated, and the fitness of remaining samples is inferred based on these ratings and distance to the centroid.

Since only a subset of the population is evaluated, the fitness of the remaining individuals must be estimated. This was achieved based on the assumption that mixes close to one another share many common attributes and are perceptually similar. The primary method of inferring the fitness of an unevaluated mix was to use the distance to the evaluated mix (the mix closest to the cluster centroid). Each mix within a cluster is awarded the same fitness as the evaluated representative and then an offset is subtracted, proportional to the distance from the centroid $[18,19]$.

\subsection{Genetic Operations}

In this example, while clustering takes place in $\mathbb{R}^{n}$, all genetic operations are performed in $\mathbb{S}^{n-1}$. This ensures that the offspring produced by crossover and mutation are always on the hypersphere in $\mathbb{R}^{n}$. Prior to genetic operations, the real-valued coordinates on $\mathbb{S}^{n-1}$ were first converted to binary strings as follows. When the values of $g$ are positive, the range of $\Phi$ is from 0 to $2 \pi$. To convert to a binary representation, first the range is re-scaled to $[0,1]$ then multiplied by $2^{q}-1$, where $q$ is the number of bits used in the binary representation. This has a range of $\left[0,2^{q}-1\right]$. In this example, $q=7$, allowing 128 levels for each variable. As an individual in the population is comprised of $d=n-$ 1 coordinates, the values of each individual dimension were converted to a $q$-bit binary string and then concatenated to form the complete parameter vector [20].
Raw fitness values are scaled according to Eq. (1), where $r$ is the rank of the individual, when sorted by fitness [15]. The result is a set of scaled fitness values in the range $[0,1]$. This has the following advantages: a) ensures that fitness values are positive, b) ensures that the range of fitness in each generation is equal, and c) prevents the emergence of "superindividuals," whose fitness is so much higher than others as to dominate the competition in breeding.

$$
f_{\text {scaled }}=\frac{1}{\sqrt{r}}
$$

A proportion of the population automatically survives to the next generation. These individuals are referred to as elites. In this case, the individuals with highest fitness are carried forward. This ensures that high-fitness solutions are not lost by the processes of crossover and mutation.

The crossover function (XO) is important because it promotes diversity in the population of solutions, helping to prevent the algorithm getting stuck in local minima. A number of alternative crossover functions were tested in order to choose the most suitable for this problem.

The performance of the uniform XO was improved over the single-point $\mathrm{XO}$, measured under the criteria of diversity of the resulting population. This allowed the population to better explore the space and increases the likelihood of convergence towards an optimal, rather than a local, solution.

Individual solutions also undergo mutation, which promotes diversity in the population. In this case, a fraction of the total bits in each solution is randomly chosen to undergo mutation. For each of these the value is changed from a 0 to a 1 or vice-versa. The greater this fraction the more noticeable the mutation.

\subsection{Stop Criteria and Choosing the Optimal Mix}

The most simple criteria would be to stop after a fixed number of generations. Alternatively, evolution could cease once the population has converged towards a sufficiently small region of the solution space. Here, it was more appropriate to use a fixed number of generations, to keep the duration of subjective tests to a predictable timescale. It is also possible that, by using the latter method, the system would not always converge. Typically, in evolutionary algorithms, the best solution is considered to be the solution with the highest fitness. There are a number of reasons why this approach is not suitable here. 


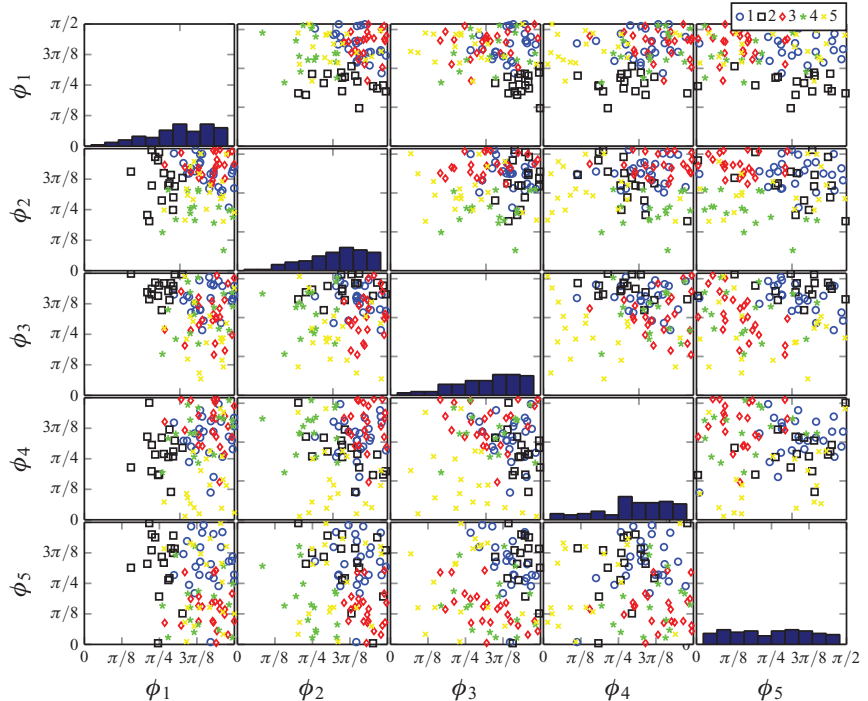

(a) Population at generation 1

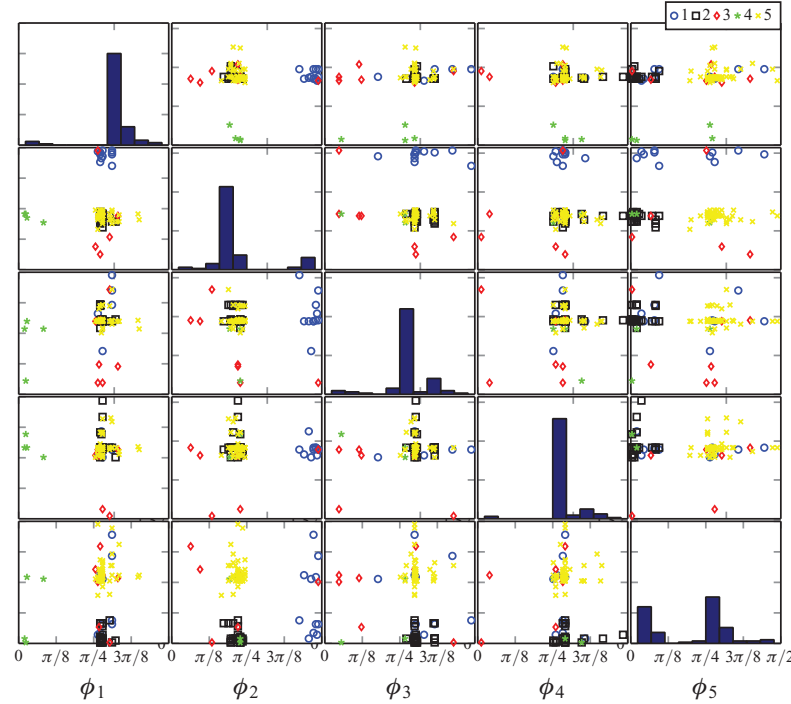

(b) Population at generation 10

Fig. 3. Population of mixes taken from one of the evaluation sessions. Symbols/colors represent the five clusters, as described in Sec. 2.2. The histograms along the main diagonal show the distribution of each $\phi$ term, and all have equal $y$-scale $(0 \rightarrow 100)$. These plots show that, while mixes are initially distributed uniformly on the hypersphere, there are noticeable regions of convergence after ten generations.

1. Since fitness was subtracted in proportion to distance from the evaluated individuals, the individual with the highest fitness will always be one of the directlyevaluated sub-population, while that might not be the case in reality.

2. Many problems that can be addressed by Interactive Evolutionary Algorithms are perceptual and as such do not require exact solutions but rather seek to identify an area of the solution space in which many good solutions exist that are perceptually similar [16]. For example, in an audio mixing problem there is a limit to the precision required when determining some metadata values. For example, small adjustments in the gain of individual tracks might not be reliably perceived.

If the population converges on a small region of the solution space, the centroid of the final population is an appropriate choice for the optimal solution, or "best" mix. Determining this point employed kernel density estimation (KDE) methods. Two methods were tested here: multiple univariate $\mathrm{KDE}$, where the density of the population is evaluated separately for each dimension, and multivariate $\mathrm{KDE}$, where the density of the population is determined in the multivariate space. The results from both methods were compared and show a high level of agreement [9]. The univariate method was used in order to reduce computation time.

\section{EVALUATION}

The aim of the work in this section is to ascertain how users interact with the system and whether or not it can be considered useful. The following are the research questions pertaining to this section.
1. What are the median loudness levels of instruments when mixed using this system?

2. How does this compare to a more traditional, faderbased approach?

3. How is the user experience evaluated, qualitatively, by the user?

The first two questions relate to the results found in the literature, indicating that voice is prioritized in music/audio mixes and some consensus is observed regarding the relative levels of drums and bass in music [9, 21]. Should similar levels and distributions of track gain be found then it could be said that the proposed system does not prohibit the user from finding the type of mix they would create with a traditional system.

In addition to finding the types of mixes that are created with the system, it is important to determine the nature of the user-experience. The third question seeks to identify if a user is likely to encounter difficulty with using the system. To answer these questions, an experiment was devised in which a number of participants were given the chance to use the system to create their desired mix of a specific song, and to report on their experience of the system. The experiment took place in the ITU-R BS.1116 compliant listening room at the University of Salford. A single loudspeaker (Genelec 8020a) was used, positioned centrally, at a distance of $1.4 \mathrm{~m}$ from the listening position. Participants were free to adjust the playback level during their evaluation of generation \#1 but not thereafter. The number of participants who took part in this experiment was 14 , most of whom had previously participated in previous audio-mixing tasks [9] and were considered to be sufficiently familiar with level-balancing of a number of audio signals. Furthermore, all were either postgraduate or undergraduate students in audio and acoustics programs, or active researchers in these areas. 

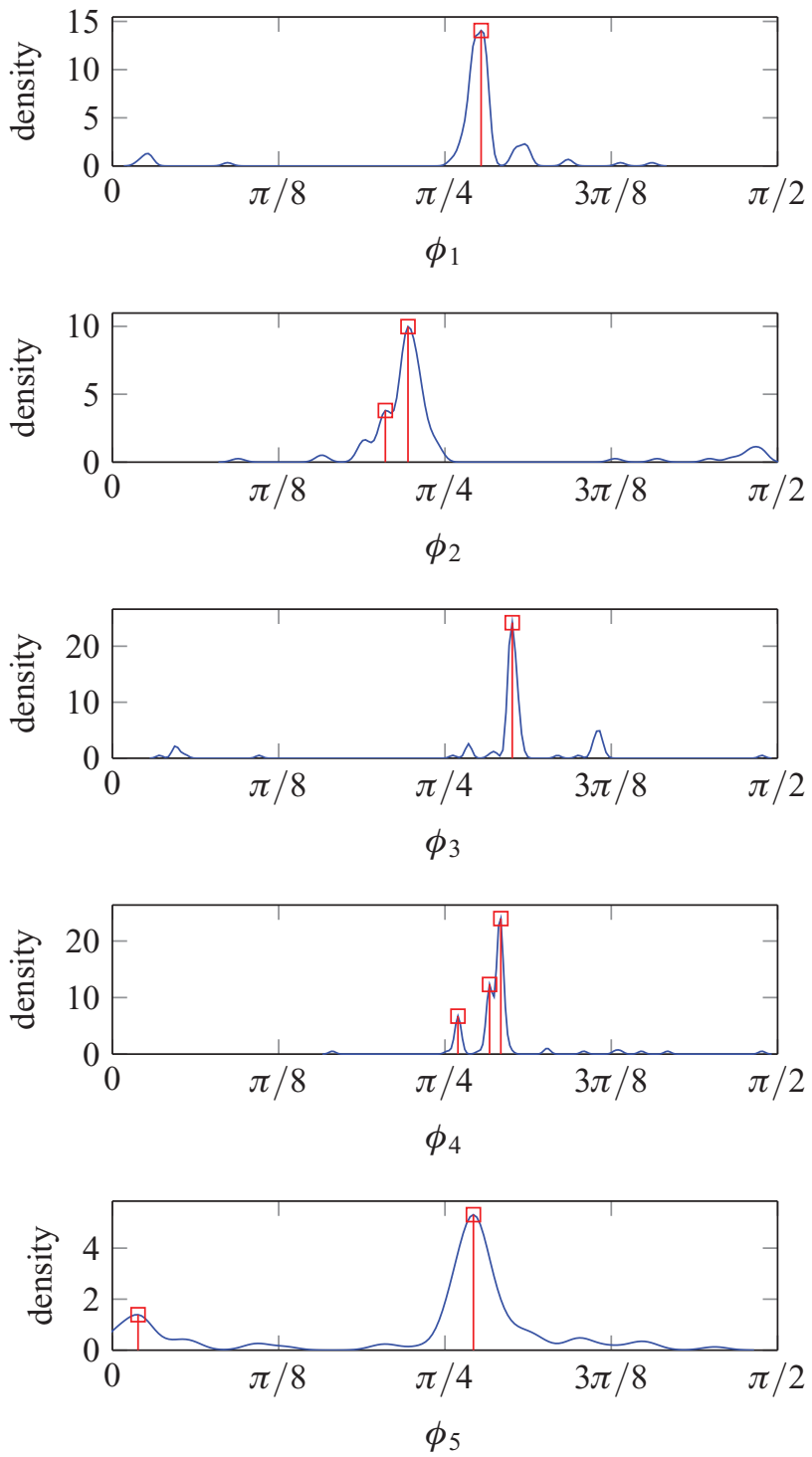

Fig. 4. Univariate KDE result from one of the sessions. Peaks in each distribution are marked. Taking the greatest peak in each provides a 5-element $\Phi$ vector (on $\mathbb{S}^{5}$ ) which is transformed to a 6 -element gain vector (in $\mathbb{R}^{6}$ ).

The only visual information presented to the user was a simple GUI to gather ratings of mixes and to provide a progress update at the end of each generation. Consequently, the user needed to rely solely on auditory perception and would not be able to mix based on visual or tactile stimuli, such as with a fader-based system. When rating mixes, participants were advised that a rating of 10/10 represented their ideal mix, while a rating of $1 / 10$ is a mix most far from ideal, in any of the many ways that this might be possible. Over all 14 participants, the median amount of time taken to evaluate 10 generations ( 50 mixes) was 11 minutes 17 seconds. As a mix deemed to be poor can be evaluated rather quickly, this short duration was not unexpected.

Upon completing 10 generations the optimal mix was estimated using the univariate KDE method (see Fig. 4). This mix was then played back to the user for informal evaluation but was not rated quantitatively.

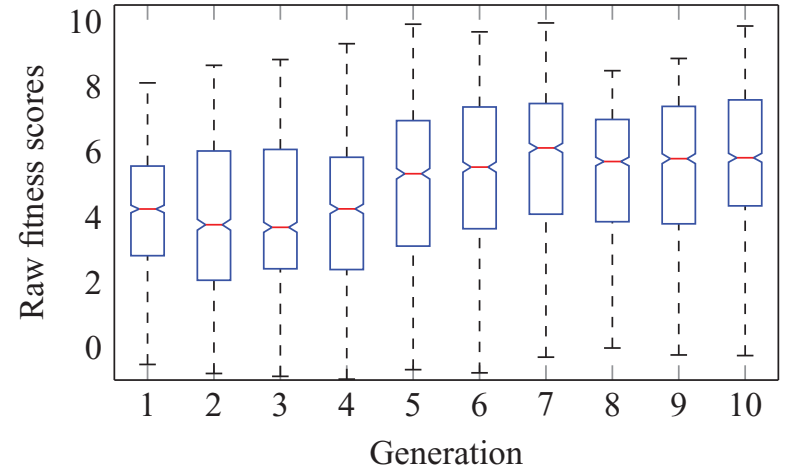

Fig. 5. Boxplot showing the raw fitness scores per generation for all 14 participants' sessions (1,400 mixes per generation).

Fig. 5 shows the distribution of raw fitness scores per generation when data from all participants was combined. As desired, the fitness of the population typically increased as the system evolves. A few additional observations can be made from this plot.

1. As the initial population is uniformly distributed on the hypersphere, there is likely to be a variety of mixes, rated good and bad. Since gen \#2 represents the first evolved generation it is credible that the median fitness may drop initially.

2. As anticipated, the fitness increases over the duration of the session, mostly between generations 3 and 7. This indicates that once the system has identified an optimum point based on user ratings, after a few generations of searching, it slowly begins to converge.

3. This convergence reaches a saturation point at generation 7 as no regular improvement in median fitness is observed from here on.

It is important to note that while the best mixes in a given generation are passed on to the next generation (as "elite" children), they may not survive another generation. This is due to the fact that the inferred fitness is always determined by subtracting an offset from the rated subset. The best mix in a given generation is therefore one that was part of the rated subset. Once the system completed 10 generations of user-evaluation and evolution, the univariate KDE method was used to determine that participant's supposed ideal mix.

\subsection{Survey Responses}

At the end of each "mixing" exercise, the user was played the "best" mix and provided with a questionnaire in order to assess the interaction between the user and the system. The first 10 questions were from the System Usability Scale (SUS), a short survey designed to gather information of a system's usability [22]. Additional questions were devised by the authors as more directly related to audio mixing systems and this particular experiment. The list of statements is shown in Table 3. For each the user chose a response on 
Table 2. Comparision of levels. Fader results are from [9], where Faders(all) pertains to the entire experimental data from that study and Faders(LS.sc) is the subset of results matching the conditions in this, IGA, study (using loudspeakers and the song "Sister Cities" by Hop Along).

\begin{tabular}{lccc}
\hline & \multicolumn{3}{c}{ Median Level (LUFS) } \\
\cline { 2 - 4 } Track & IGA & Faders(LS.sc) & Faders(all) \\
\hline Vox & -2.72 & -2.30 & -2.85 \\
Gtr & -10.84 & -8.89 & -8.56 \\
Bass & -10.37 & -10.43 & -10.46 \\
Drums & -7.62 & -8.33 & -8.11 \\
Snare & -14.57 & & \\
Kick & -16.69 & & \\
OH & -12.79 & & \\
\hline
\end{tabular}

a 5-point Likert scale, marked at the extremes by "strongly disagree" and "strongly agree."

High scores on odd numbered questions indicate a positive impression of system usability, as do low scores on even-numbered questions. Scoring of the questionnaire results is as follows: for odd items, subtract one from the user response. For even-numbered items, subtract the user responses from 5 . This scales all values from 0 to 4 (with four being the most positive response). Sum the converted responses for each user and multiply the total by 2.5. This converts the range of possible values from 0 to 10 .

Table 3 shows the mean of the converted scores for each item. Note that the score shown for items 1 to 10 is the mean positivity (from 0 to 4 ), not the mean of the raw scores (i.e., not the level of agreement with the statement). For items 11 to 17 the score shown is the mean level of agreement with the statement. Across all users, the median SUS score for the system is 90 , while the range was 75 to 95 . This score by itself does not offer much insight without other systems to compare to. [23] analyzed the SUS scores from a variety of different systems and found the average SUS score from over 200 studies to be 70 . This suggests that the proposed system is highly usable.

\section{DISCUSSION}

\subsection{Optimal Levels}

A comparison between the median track levels obtained in this experiment and in a fader-based experiment in the same location is shown in Table 2. This reveals that differences found between the two methods are small. The largest difference is that the guitar was typically set quieter using the IGA system, by about 2 LU. The level of the vocals in the IGA experiment is closer to the Faders(all) level than Faders(LS.sc), indicating that this level may generalize well to other songs. A precise match between experiments would have been surprising, especially considering the IGA method only approximates the user's ideal mix in the final KDE stage. That said, the close match for vocals, bass, and drums (to a slightly lesser extent) indicates the success of the IGA method. From this it may be claimed with some
Table 3. Survey results for IGA mixer, showing the mean and standard deviation of the data.

\begin{tabular}{|c|c|c|c|}
\hline \# & Statement & $\begin{array}{l}\text { avg. } \\
\text { positivity }\end{array}$ & std. dev \\
\hline 1 & $\begin{array}{l}\text { I think that I would like to use this } \\
\text { system frequently. }\end{array}$ & 2.92 & 0.64 \\
\hline 2 & $\begin{array}{l}\text { I found the system unnecessarily } \\
\text { complex. }\end{array}$ & 3.69 & 0.48 \\
\hline 3 & $\begin{array}{l}\text { I thought the system was easy to } \\
\text { use. }\end{array}$ & 3.92 & 0.28 \\
\hline 4 & $\begin{array}{l}\text { I think I would need the support of } \\
\text { a technical person to be able to } \\
\text { use this system. }\end{array}$ & 3.77 & 0.44 \\
\hline 5 & $\begin{array}{l}\text { I found the various functions in } \\
\text { this system were well integrated. }\end{array}$ & 3.54 & 0.66 \\
\hline 6 & $\begin{array}{l}\text { I thought there was too much } \\
\text { inconsistency in this system. }\end{array}$ & 3.15 & 0.99 \\
\hline 7 & $\begin{array}{l}\text { I would imagine that most people } \\
\text { would learn to use this system } \\
\text { very quickly. }\end{array}$ & 3.54 & 0.66 \\
\hline 8 & $\begin{array}{l}\text { I found the system very } \\
\text { cumbersome to use. }\end{array}$ & 3.38 & 0.65 \\
\hline 9 & $\begin{array}{l}\text { I felt very confident using the } \\
\text { system. }\end{array}$ & 3.54 & 0.66 \\
\hline 10 & $\begin{array}{l}\text { I needed to learn a lot of things } \\
\text { before I could get going with } \\
\text { this system. }\end{array}$ & 3.46 & 0.97 \\
\hline
\end{tabular}

\begin{tabular}{|c|c|c|c|}
\hline \multicolumn{4}{|c|}{ avg. score } \\
\hline 11 & $\begin{array}{l}\text { I felt in control of the mixing } \\
\text { process. }\end{array}$ & 2.69 & 0.95 \\
\hline 12 & $\begin{array}{l}\text { I thought the loudness of samples } \\
\text { was consistent. }\end{array}$ & 3.85 & 0.55 \\
\hline 13 & $\begin{array}{l}\text { I felt the mixes got better over } \\
\text { time. }\end{array}$ & 3.62 & 0.77 \\
\hline 14 & $\begin{array}{l}\text { I found the interface to be } \\
\text { physically demanding. }\end{array}$ & 1.31 & 0.85 \\
\hline 15 & $\begin{array}{l}\text { I thought the loudness of samples } \\
\text { was suitable. }\end{array}$ & 4.31 & 0.63 \\
\hline 16 & $\begin{array}{l}\text { I found the interface to be } \\
\text { mentally demanding. }\end{array}$ & 1.31 & 0.63 \\
\hline 17 & $\begin{array}{l}\text { I felt the test environment was } \\
\text { comfortable. }\end{array}$ & 4.77 & 0.44 \\
\hline
\end{tabular}

confidence that the IGA method is capable of creating a range of mixes similar to that which would be created using the conventional fader-based approach but using a very different and much less complex interface.

\subsection{Usability}

The statement that received the least positive response was \#1 ("I think that I would like to use this system frequently"). Initially, this particular observation seems to contradict the overall high score that users awarded the system. However, while it is the least positive response, the mean score is 2.92 on a scale of 0 to 4 , suggesting a result that is still rather positive. However, it is important to realize that the users would have been comparing the system to a more conventional audio mixing system and it is likely that some preference would typically lie with a known, more "hands on" method. Somewhat supporting this suggestion, the next least positive statement was \#6 ("I thought there 
was too much inconsistency in this system"). This seems to indicate that difficulties experienced by users were due to lack of direct, explicit control over the parameters of the mix, as further corroborated by the results pertaining to statement \#11 ("I felt in control of the mixing process"). When asked whether the system was either physically or mentally demanding, users typically responded that neither was the case, suggesting the system has a low level of userburden. Achieving its goal of low or inexistent physical burden affords a high level of accessibility. From the SUS items, the statement obtaining the most positive response was \#3 ("I thought the system was easy to use"). Importantly, users generally felt that mixes got better over time, as desired.

\subsection{Applications}

The system described in this paper allows a method of audio which relies only on audition, as it features a minimal amount of visual or tactile stimuli. Users described the system has having low levels of physical or mental burden. Consequently, this system could act as a mixing assistant to a visually impaired audio engineer, or be tailored to suit a range of accessibility requirements, such as where mobility is impaired. It is hoped that further research into such interfaces could widen participation in audio engineering and broadcast careers.

In this paper the user of the system partakes in active fitness evaluation, carefully considering the qualities of each presented solution, thus allowing the all-important human decision into the final outcome. One challenge moving forward will be to complement this with other forms of fitness evaluation, where the rating of solutions is embedded in some other process such that the user is not aware of the system's learning process. One such form could be achieved through implementing bio-physiological response into the interaction loop [24]. This would allow for increased personalization of object-based content, where the renderer adapts to the requirements of the user in an unobtrusive manner.

\subsection{Further Work}

While a typical genetic algorithm relies on an objective fitness function, this is difficult to implement for a subjective task such as audio-mixing. In the case of a televised/radio drama, measures such as speech intelligibility could be used as an objective function, however a variety of constraints would be necessary, as intelligibility can easily be maximized by simply muting all other objects.

With each learning session, the system has the potential to adapt further. By associating the evolution of the solution with the measured signal features of the input audio tracks, the system could further learn general traits of audio mixing. It has been shown that in large collections of music-mixes, there is noticeable central tendency in the distributions of audio signal features [17]. This could be used to provide objective rules to help constrain the system in addition to the guidance of the user.
Whether or not this is desired is another issue. In this paper the aesthetic proposed is one where the system makes no prior assumptions of the process. Earlier attempts at automatic rendering have perhaps had an over-reliance on prior assumptions and so-called best-practice mixing techniques. Combining both strategies - adapting to a specific user while also learning best-practice from a collection of users-will be a challenge in further development of this and related systems.

\section{CONCLUSIONS}

This paper has described a user-guided rendering system, using an interactive genetic algorithm, which could be utilized in object-based broadcast or in a more traditional production environment. While this paper deals only with audio levels, addition of panning and equalization is possible. When using the proposed system in a simple musicmixing task, participants were able to create a range of mixes comparable to those made using the conventional fader-based system. This suggests that the system is not an obstacle to the creation of desired content and does not impose noticeable limits on what content can be created. The system was considered to be highly usable. Both physical and mental demands were reported to be low. Consequently it is predicted that the system would be suitable for a variety of applications where physical interaction is to be kept low.

\section{REFERENCES}

[1] M. Armstrong, M. Brooks, A. Churnside, M. Evans, F. Melchoir, and M. Shotton, "Object-Based Broadcasting-Curation, Responsiveness and User Experience," IET Conference Proceedings, no.1, pp. 12.2-12.2 (2014 Jan.), doi: 10.1049/ib.2014.0038.

[2] J. Scott and Y. E. Kim, "Instrument Identification Informed Multi-Track Mixing," presented at the 14th International Society for Music Information Retrieval Conference (ISMIR 2013) (2013).

[3] B. De Man, Towards a Better Understanding of Mix Engineering, Ph.D. thesis, Queen Mary University of London (2017).

[4] D. M. Ronan, H. Gunes, and J. D. Reiss, "Analysis of the Subgrouping Practices of Professional Mix Engineers," presented at the 142nd Convention of the Audio Engineering Society (2017 May), convention paper 9700.

[5] D. Ronan, J. D. Reiss, and H. Gunes, "An Empirical Approach to the Relationship between Emotion and $\mathrm{Mu}-$ sic Production Quality," arXiv preprint arXiv:1803.11154 (2018).

[6] M. Armstrong, "From Clean Audio to Object Based Broadcasting," BBC R\&D White Paper, WHP, vol. 324 (2016).

[7] L. Ward, B. Shirley, Y. Tang, and W. J. Davies, "The Effect of Situation-Specific Non-Speech Acoustic Cues on the Intelligibility of Speech in Noise," Proc. Interspeech 2017, pp. 2958-2962 (2017), doi: 10.21437/ interspeech.2017-500. 
[8] A. Wilson and B. M. Fazenda, "Perception of Audio Quality in Productions of Popular Music," J. Audio Eng. Soc., vol. 64, pp. 23-34 (2016 Jan./Feb.), doi: https://doi. org/10.17743/jaes.2015.0090.

[9] A. Wilson, Evaluation and Modelling of Perceived Audio Quality in Popular Music, towards Intelligent Music Production, Ph.D. thesis, University of Salford (2017).

[10] A. Wilson, R. Loughran, and B. M. Fazenda, "On the Suitability of Evolutionary Computing to Developing Tools for Intelligent Music Production," presented at the 3rd Workshop on Intelligent Music Production (WIMP 2017) (2017).

[11] A. Wilson and B. M. Fazenda, "Navigating the Mix-Space: Theoretical and Practical LevelBalancing Technique in Multitrack Music Mixtures," presented at the Sound and Music Computing Conference (2015 July).

[12] A. Wilson and B. M. Fazenda, "Populating the Mix Space: Parametric Methods for Generating Multitrack Audio Mixtures," Applied Sciences, vol. 7, no. 12, p. 1329 (2017), doi: 10.3390/app7121329.

[13] P. D. Pestana, J. D. Reiss, and A. Barbosa, "Loudness Measurement of Multitrack Audio Content Using Modifications of ITU-R BS. 1770," presented at the 134th Convention of the Audio Engineering Society (2013 May), convention paper 8813 .

[14] K. Hornik, I. Feinerer, M. Kober, and C. Buchta, "Spherical k-Means Clustering," J. Stat. Software, vol. 50, no. 10, pp. 1-22 (2012), doi: 10.18637/jss.v050.i10.

[15] D. Goldberg, K. Deb, and B. Korb, "Messy Genetic Algorithms: Motivation, Analysis, and First Results," Complex Systems, no. 3, pp. 493-530 (1989).

[16] H. Takagi, "Interactive Evolutionary Computation: Fusion of the Capabilities of EC Optimization and
Human Evaluation," Proceedings of the IEEE, vol. 89, no. 9, pp. 1275-1296 (2001), doi: 10.1109/5.949485.

[17] A. Wilson and B. Fazenda, "Variation in Multitrack Mixes: Analysis of Low-Level Audio Signal Features," J. Audio Eng. Soc., vol. 64, pp. 466-473 (2016 Jul./Aug.), doi: 10.17743/jaes.2016.0029.

[18] J.-Y. Lee and S.-B. Cho, "Sparse Fitness Evaluation for Reducing User Burden in Interactive Genetic Algorithm," IEEE International Fuzzy Systems Conference Proceedings (FUZZ-IEEE'99), pp. 998-1003 vol. 2 (1999), doi: 10.1109/FUZZY.1999.793088.

[19] H.-S. Kim and S.-B. Cho, "Application of Interactive Genetic Algorithm to Fashion Design," Engineering Applications of Artificial Intelligence, vol. 13, no. 6, pp. 635-644 (2000), doi: 10.1016/S0952-1976(00)000452.

[20] A. H. Wright, "Genetic Algorithms for Real Parameter Optimization," in Foundations of Genetic Algorithms, vol. 1, pp. 205-218 (Elsevier, 1991), doi: 10.1016/b978-008-050684-5.50016-1.

[21] B. De Man, B. Leonard, R. King, and J. D. Reiss, "An Analysis and Evaluation of Audio Features for Multitrack Music Mixtures," 15th International Society for Music Information Retrieval Conference (ISMIR 2014), pp. 137-142 (2014).

[22] J. Brooke, et al., "SUS-A Quick and Dirty Usability Scale," Usability Evaluation in Industry, vol. 189, no. 194, pp. 4-7 (1996).

[23] A. Bangor, P. T. Kortum, and J. T. Miller, "An Empirical Evaluation of the System Usability Scale," Intl. J. Human-Computer Interaction, vol. 24, no. 6, pp. 574-594 (2008), doi: 10.1080/10447310802205776.

[24] D. A. H. Williams, D. T. Murphy, and B. Fazenda, "Towards Bio-Responsive Control for Music," presented at the DMRN+12 Digital Music Research Network (2017). 


\section{THE AUTHORS}

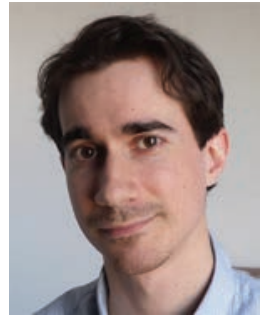

Alex Wilson

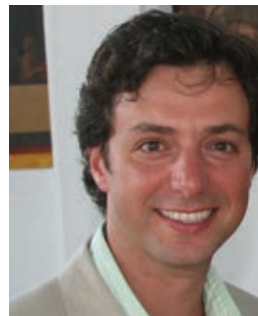

Bruno M. Fazenda

Alex Wilson is a postdoctoral researcher at the University of Salford, investigating the perception of object-based audio reproduction systems. He completed a Ph.D. at the same institution in 2017, investigating the perception of audio quality in music production. He is interested in the intersection of audio technologies and human decision-making and has published works relating to psychoacoustics, digital signal processing, music production, accessibility, scientometrics, and social network analysis. He is a committee member of the AES Ireland section, since it's foundation in 2019.

Bruno Fazenda received a B.Sc. (1st Hons.) degree in audio technology in 1999 and a Ph.D. degree in 2004 for

his thesis on the perception of room modes, both from the University of Salford, UK. He worked for a short while as a research fellow with a Marie Curie research fellowship at the Danish Technical University before becoming a lecturer in the UK. He now lectures in the acoustics and audio area at the University of Salford. His research interests in acoustics, psychoacoustics, multimodal perception in virtual realities and archaeoacoustics, focussing particularly on the assessment of how an acoustic environment, technology or psychological state impacts on the perception of sound. He is also a keen student on aspects of human evolution, perception, and brain function. He is a member of the Audio Engineering Society. 